\author{
Caroline Fässler \\ Eva Arrigoni \\ Koen Venema \\ Valeria Hafner \\ Fred Brouns \\ Renato Amadò
}

\section{Digestibility of resistant starch containing preparations using two in vitro models}

Received: 23 March 2006

Accepted: 28 August 2006

Published online: 11 October 2006

C. Fässler · E. Arrigoni ( $\square)$

V. Hafner $\cdot$ R. Amadò

Institute of Food Science and Nutrition

ETH Zurich, LFO D28

Schmelzbergstr. 9

8092 Zürich, Switzerland

Tel.: +41-44/632-3374

Fax: +41-44/632-1123

E-Mail: eva.arrigoni@ilw.agrl.ethz.ch

K. Venema

TNO Quality of Life

Dept. of Physiological Sciences

Zeist, The Netherlands

F. Brouns

Cerestar-Cargill R\&D Centre

Vilvoorde, Belgium

F. Brouns

Nutrition and Toxicology Research

Institute

Maastricht University

Maastricht, The Netherlands
Abstract Background Resistant starch (RS) is known for potential health benefits in the human colon. To investigate these positive effects it is important to be able to predict the amount, and the structure of starch reaching the large intestine. Aim of the study The aim of this study was to compare two different in vitro models simulating the digestibility of two RS containing preparations. Methods The substrates, high amylose maize (HAM) containing RS type 2, and retrograded long chain tapioca maltodextrins (RTmd) containing RS type 3 were in vitro digested using a batch and a dynamic model, respectively. Both preparations were characterized before and after digestion by using X-Ray and DSC, and by measuring their total starch, RS and protein contents. Results Using both digestion models, 60$61 \mathrm{~g} / 100 \mathrm{~g}$ of RTmd turned out to be indigestible, which is very well in accordance with $59 \mathrm{~g} / 100 \mathrm{~g}$ found in vivo after feeding RTmd to ileostomy patients. In contrast, dynamic and batch in vitro digestion experiments using HAM as a substrate led to $58 \mathrm{~g} / 100 \mathrm{~g}$ and $66 \mathrm{~g} / 100 \mathrm{~g}$ RS recovery. The degradability of HAM is more affected by differences in experimental parameters compared to RTmd. The main variations between the two in vitro digestion methods are the enzyme preparations used, incubation times and mechanical stress exerted on the substrate. However, for both preparations dynamically digested fractions led to lower amounts of analytically RS and a lower crystallinity. Conclusions The two in vitro digestion methods used attacked the starch molecules differently, which influenced starch digestibility of HAM but not of RTmd.

Key words resistant starch type 2 - resistant starch type 3 in vitro digestion DSC - X-ray

\section{Introduction}

Resistant Starch (RS) is defined as starch that is not absorbed in the small intestine of humans. Three different types of RS were defined by Englyst et al. [1]. Type 1 is defined as physically inaccessible starch, type 2 (RS2) as native starch granules and type 3
(RS3) as retrograded starch. More recently, a fourth type of RS has been classified, comprising chemically modified starches $[2,3]$. RS is claimed to be a good substrate for colonic fermentation and to be beneficial because of its high ratio of butyrate production, which may play a major role in the prevention of colon cancer, as shown in several studies on animal models 
$[4,5]$. The fermentation products from RS are known to lower the $\mathrm{pH}$ in the colon which leads to less production, and/or accumulation of potentially harmful by-products of protein fermentation, for example, ammonia or phenols which may promote tumorigenesis [6]. To estimate potential health benefits of RS it is important to be able to predict its behavior in the human gastrointestinal tract, in particular the amount and the structure of starch reaching the large intestine. The digestion of food and absorption of nutrients are spatiotemporal and dynamic processes involving complex enzymatic systems and transport reactions. Thus, the simulation of all these biochemical and physiological events in a single model is illusive. Nevertheless, several in vitro digestion methods, from basic batch systems to sophisticated dynamic models have been developed in order to quantify digestibility of food [7-9]. A realistic approach implies a well-defined system that takes into account the specific contributions of oral, gastric and intestinal digestion. Advantages of using in vitro instead of in vivo models are low costs, relatively easy performance, no limitations by ethical constraints, and the possibility to compare different substrates when applying standardized conditions. Therefore, the aim of this study was to compare a rather simple and a more sophisticated in vitro model as well as the Megazyme RS method to determine the indigestible fractions of two RS preparations containing RS2 and RS3. The digestion residues obtained by the two models were compared and characterized. Furthermore, the RS3 fractions were compared with ileostomy effluents obtained in an in vivo study [10].

\section{Materials and methods}

\section{Substrates}

The RS3 containing carbohydrate source, C 2 Acti$\operatorname{star}^{\circledR}$, is a retrograded long chain maltodextrin product obtained after partial enzymatic hydrolysis, and subsequent retrogradation of tapioca starch (RTmd), produced according to United States Patent 6043229 [11] and obtained from Cerestar-Cargill (Vilvoorde, Belgium). Physico-chemical characteristics of RTmd were described recently by Pohu [12]. The RS2 containing carbohydrate source is native high amylose maize (HAM) starch and was obtained from Cerestar International (Neuilly-sur-Seine, France).

\section{Batch in vitro digestion model}

The batch model used is a three step incubation at $37^{\circ} \mathrm{C}$ simulating the digestion in mouth, stomach and small intestine. The method was carried out as described by Lebet et al. [7] and modified by Jörger [13]. A sample of $30 \mathrm{~g}$ substrate was suspended in $500 \mathrm{ml}$ phosphate buffer $\left(20 \mathrm{mM}, \mathrm{pH} 6.9, \mathrm{Na}_{2} \mathrm{HPO}_{4} 1.42 \mathrm{~g} / \mathrm{l}\right.$, $\left.\mathrm{KH}_{2} \mathrm{PO}_{4} 1.36 \mathrm{~g} / \mathrm{l}, \mathrm{NaCl} 0.58 \mathrm{~g} / \mathrm{l}\right)$, and incubated stepwise with (a) $0.5 \mathrm{ml}$ human salivary $\alpha$-amylase solution (Sigma A1031, Buchs, $\mathrm{CH}, 10 \mathrm{mg} / \mathrm{ml}$ in $\mathrm{CaCl}_{2}$ $1 \mathrm{mM}$ ) at $\mathrm{pH} 6.9$ for $15 \mathrm{~min}$, (b) $1.25 \mathrm{ml}$ porcine pepsin suspension (Sigma P7012, $1 \mathrm{mg} / \mathrm{ml}$ in $\mathrm{NaCl}$ $9 \mathrm{~g} / \mathrm{l}$ ) at $\mathrm{pH} 2.0$ for $30 \mathrm{~min}$ and (c) $10 \mathrm{ml}$ porcine pancreatin (Sigma P7545, $0.5 \mathrm{mg} / \mathrm{ml}$ in $\mathrm{CaCl}_{2} 25 \mathrm{mM}$ ) at $\mathrm{pH} 6.9$ for $3 \mathrm{~h}$ in the presence of $12 \mathrm{~g}$ bovine bile (Sigma B8381). Degradation products were removed by dialysis (Servapor 44146, Serva Feinbiochemica $\mathrm{GmbH} \&$ Co., Heidelberg, Germany; cut-off 12$14 \mathrm{kDa}$ ) overnight under continuous movements against running deionized water $<20^{\circ} \mathrm{C}$. The retentate was freeze-dried. Based on total starch (TS) determinations in the starting materials and digestion residues, the amount of indigestible starch was calculated.

\section{Dynamic in vitro digestion model (TIM-1)}

The dynamic model used in this study has been described by Minekus et al. [8]. The model comprises four serial compartments simulating stomach, duodenum, jejunum and ileum. The in vitro digestion was performed for $6 \mathrm{~h}$ at $37^{\circ} \mathrm{C}$. A mixture of $60 \mathrm{~g}$ substrate, $180 \mathrm{~g}$ electrolyte solution $(\mathrm{NaCl} 5 \mathrm{~g} / \mathrm{l}, \mathrm{KCl}$ $\left.0.6 \mathrm{~g} / \mathrm{l}, \mathrm{CaCl}_{2} \cdot 2 \mathrm{H}_{2} \mathrm{O} 0.3 \mathrm{~g} / \mathrm{l}, \mathrm{NaHCO}_{3} 0.6 \mathrm{~g} / \mathrm{l}\right), 60 \mathrm{~g}$ water, $5 \mathrm{~g}$ pepsin solution (Sigma P7012, $0.28 \mathrm{~g} / \mathrm{l}$ in solution A: $\mathrm{NaCl} 3.1 \mathrm{~g} / \mathrm{l}, \mathrm{KCl} 1.1 \mathrm{~g} / \mathrm{l}, \mathrm{CaCl}_{2} \cdot 2 \mathrm{H}_{2} \mathrm{O}$ $\left.0.15 \mathrm{~g} / \mathrm{l}, \mathrm{NaHCO}_{3} 7.1 \mathrm{~g} / \mathrm{l}\right)$ and $5 \mathrm{~g}$ lipase solution (Rhizopus lipase, Amano Pharmaceutical Co. F-AP 15 , Ltd. Japan, $0.25 \mathrm{~g} / \mathrm{l}$ in solution A) were introduced into the gastric compartment. Computer controlled peristaltic valve pumps controlled meal transit through the individual compartments. The $\mathrm{pH}$ value was computer monitored by adding $\mathrm{HCl}(1 \mathrm{M})$ or $\mathrm{NaHCO}_{3}(1 \mathrm{M})$, respectively. In the stomach the values were preset to $\mathrm{pH} 4.5,2.8,1.8,1.7$ and 1.5 at 0,20 , 40,60 and $90 \mathrm{~min}$, respectively. In the small intestine, the $\mathrm{pH}$ was maintained at $6.5,6.8$ and 7.2 in the duodenum, jejunum and ileum, respectively. About $1 \mathrm{ml}$ trypsin solution (Sigma T4665, $2 \mathrm{~g} / \mathrm{l}$ in solution A) was added to the duodenum at the beginning of the experiment. Secretions of porcine bile $4 \mathrm{~g} / 100 \mathrm{~g}$ in water (Sigma B8631) and pancreatic solution $7 \mathrm{~g} /$ $100 \mathrm{~g}$ in water (Pancrex-V powder, Paines \& Byrne, Greenford, UK) entered the duodenal compartment at 0.5 and $0.25 \mathrm{ml} / \mathrm{min}$, respectively. The absorption of water and digestive products from the jejunal and ileal compartments was simulated using hollow-fiber devices (cut-off 5-10 kDa). Ileal effluents (indigestible fraction) were collected after 2,4 and $6 \mathrm{~h}$, pooled and 
freeze-dried. Substrate remaining in the jejunum and ileum at the end of the experiment was considered to be indigestible as well and was therefore mixed with the ileal effluent pool. TS determinations of the starting materials, the digestion residues and the remaining substrate in the model after the experiment were carried out. Starch degradation products (up to DP 7) were quantified in dialysates by high-performance anion-exchange chromatography with pulsed amperometric detection (HPAEC-PAD) in a BioLC System (Dionex Corp., Sunnyvale CA, USA) using a Carbo-Pac PA1 column and applying a sodium acetate gradient (unpublished, internal method). TS and oligosaccharide quantifications were recalculated as glucose content. The recovered glucose after the experiment (in the digestible and indigestible residues) was assumed as $100 \%$, of which the indigestible fraction is expressed as a fraction.

\section{Characterization}

Both RS preparations were characterized before and after digestion. Additionally, freeze dried RTmd fractions from an ileostomy study feeding RTmd were pooled (7 subjects) and used for characterization analyses [10].

TS was determined using the Megazyme TS assay kit (Megazyme, Co. Wicklow, Ireland) [14]. In brief, starch was hydrolyzed in two phases. In phase 1, starch was pre-treated with dimethyl-sulfoxide (DMSO), totally solubilized and partially hydrolyzed with $\alpha$-amylase. In phase 2 , dextrins were quantitatively hydrolyzed to glucose with amyloglucosidase (AMG). Glucose was subsequently determined enzymatically using the Hexokinase/Glucose-6-phosphatedehydrogenase assay according to Boehringer [15].

The RS fraction was determined using the Megazyme RS assay kit (Megazyme, Co. Wicklow, Ireland) [16]. The samples were incubated in a shaking water bath with pancreatic $\alpha$-amylase and AMG at $37^{\circ} \mathrm{C}$ for $16 \mathrm{~h}$, during which time non-resistant starch was solubilized and hydrolyzed to glucose. RS was recovered as a pellet after centrifugation, which was dissolved by stirring in $\mathrm{KOH} 2 \mathrm{M}$ and quantitatively hydrolyzed to glucose with AMG [16]. Glucose was determined as described above.

Protein was determined as sum of amino acids after acid hydrolysis and ion-exchange chromatography with post-column ninhydrin detection [17].

Wide-angle X-Ray powder diffraction (X-Ray) measurements were performed using a Scintag PADX Diffractometer (PANAlytical, Almelo, NL) operating at $45 \mathrm{~mA}$ and $40 \mathrm{kV}$ at an angular range of $2 \theta$ from 1 to $40^{\circ}$ with a step size of $0.03^{\circ}$. Counting time was $5 \mathrm{~s}$ on each step.

Differential scanning calorimetry (DSC) measurements were performed using the Thermal Analyst system 2000 (DSC 2910, TA Instument Ltd, Newcastle, UK). Samples at a starch:water ratio of 1:4 were prepared and heated from $4^{\circ} \mathrm{C}$ to $200^{\circ} \mathrm{C}$ at $10^{\circ} \mathrm{C} / \mathrm{min}$, thereafter the samples were cooled at $20^{\circ} \mathrm{C} / \mathrm{min}$ to $4^{\circ} \mathrm{C}$ and heated again at the same heating rate. All the DSC results were evaluated from the mean of three separate determinations for each sample, unless stated otherwise.

\section{Statistical analysis}

Statistical analyses were carried out using SPSS, version 12.0.1 for Windows (one-way ANOVA and PostHoc Tukey). Differences were considered to be significant at $P<0.05$.

\section{Results}

\section{Composition and digestibility of RS containing preparations}

In Table 1 the average TS contents and indigestible starch fractions of RTmd and HAM are shown. All data are mean values of at least five repetitions. Data resulting from the dynamic and the batch model are based on the TS content before and after digestion. Both preparations contain identical amounts of TS. The two in vitro digestion models as well as the Megazyme RS method which is accepted as the official AOAC method for RS determination, led to similar
Table 1 Average total starch (TS) content of retrograded tapioca maltodextrins (RTmd) and high amylose maize starch (HAM) and their indigestible starch fractions obtained by dynamic digestion, batch digestion and the Megazyme resistant starch (RS) method (mean values \pm SD)

\begin{tabular}{llll}
\hline Sample & TS $(\mathrm{g} / 100 \mathrm{~g} \mathrm{dm})$ & Digestion method & Indigestible starch fraction $(\mathrm{g} / 100 \mathrm{~g} \mathrm{TS} \mathrm{dm})$ \\
\hline RTmd & $94.3 \pm 1.8(n=17)$ & Dynamic & $59.6 \pm 2.1(n=6)^{\mathrm{a}, \mathrm{b}}$ \\
& & Batch & $60.6 \pm 0.9(n=5)^{\mathrm{a}}$ \\
& & Megazyme & $57.7 \pm 2.9(n=12)^{\mathrm{b}}$ \\
HAM & $94.3 \pm 1.1(n=7)$ & Dynamic & $57.5 \pm 4.7(n=5)^{\mathrm{d}}$ \\
& & Batch & $65.8 \pm 3.5(n=10)^{\mathrm{c}}$ \\
& & Megazyme & $54.8 \pm 5.0(n=17)^{\mathrm{d}}$ \\
\hline
\end{tabular}

Means with the same letters are not significantly different (Tukey $P<0.05$ ) 
amounts of indigestible RS3 fractions. Digestibility of RS2 was similar using the dynamic model, and the Megazyme RS method, whereas the batch model led to a lower digestibility. Expressing the RS results obtained by the Megayzme RS method based on substrate fresh weight (instead of g/100 $\mathrm{g}$ TS as shown in Table 1) yielded to $50.2 \pm 2.6$, and $45.3 \pm 4.1 \mathrm{~g} /$ $100 \mathrm{~g}$ for RTmd and HAM, respectively. This is very well in accordance with respectively, $48.3 \pm 2.8$ and $46.3 \pm 3.9 \mathrm{~g} / 100 \mathrm{~g}$ found in a collaborative study published earlier by McCleary et al. [16]. Standard deviations after HAM digestion were found to be higher compared to RTmd digestion. The batch system showed a better reproducibility than the dynamic model and Megazyme RS method for both substrates.

\section{Composition of digestion residues}

In Table 2 the average TS and RS contents of RTmd and HAM digestion residues are shown. TS quantifications of in vitro digestion residues were carried out after each single experiment in duplicate. In contrast, RS contents as well as TS in ileostomy effluents were measured in the pooled fractions only. TS contents in digestion residues turned out to be lower than in corresponding starting materials (Table 1), which are due to additional enzymes and bile added during digestion. Moreover, residues obtained from the batch digestion contained more starch than those from the dynamic model. The digestion residues of RTmd and HAM obtained from the dynamic model contained $1.5 \mathrm{~g}$ protein $/ 100 \mathrm{~g} \mathrm{dm}$ and $1.8 \mathrm{~g}$ protein $/ 100 \mathrm{~g} \mathrm{dm}$, respectively, whereas in the batch digestion residues only $0.6 \mathrm{~g}$ protein $/ 100 \mathrm{~g} \mathrm{dm}$ and $1.2 \mathrm{~g}$ protein/ $100 \mathrm{~g} \mathrm{dm}$ were determined. Bile contains large amounts of glycine that was correspondingly found in higher amounts in the dynamically digested residues $(0.2$ and $0.3 \mathrm{~g} / 100 \mathrm{~g} \mathrm{dm}$ for RTmd and HAM, respectively) compared to the batch digested residues $(0.3$ and $0.4 \mathrm{~g} / 100 \mathrm{~g} \mathrm{dm}$ for RTmd and HAM, respectively). Also other amino acids like asparagine, glutamine, alanine, proline, and leucine were found in higher amounts in dynamically digested preparations. The lower amount of TS found in the ileal effluent

Table 2 Total starch (TS) and resistant starch (RS) contents of in vitro and in vivo digestion residues of retrograded tapioca maltodextrins (RTmd) and high amylose maize starch (HAM) (mean values \pm SD)

\begin{tabular}{lll}
\hline Sample & TS $(\mathrm{g} / 100 \mathrm{~g} \mathrm{dm})$ & RS $(\mathrm{g} / 100 \mathrm{~g} \mathrm{dm})$ \\
\hline RTmd dynamic & $72.4 \pm 3.7(n=12)$ & $36.4 \pm 1.0(n=2)$ \\
RTmd batch & $88.6 \pm 0.6(n=10)$ & $61.4 \pm 1.2(n=3)$ \\
RTmd in vivo & $48.4 \pm 0.0(n=2)$ & $31.3 \pm 1.1(n=2)$ \\
HAM dynamic & $74.9 \pm 1.7(n=10)$ & $27.6 \pm 2.0(n=2)$ \\
HAM batch & $80.9 \pm 2.6(n=12)$ & $35.7 \pm 0.7(n=3)$ \\
\hline
\end{tabular}

pool compared to the in vitro digested samples is due to the dilution by additional endogenous and exogenous material such as diet derived protein, dietary fiber and fat.

It is expected that RS values close to $100 \%$ are found in the in vivo as well as the in vitro digestion residues. As shown in Table 2 lower amounts of RS $(28-62 \%)$ were found in the digestion residues. This can be explained by the fact that the digestion method carried out prior to the analytical RS determination has an influence on the starch structures leading to less resistant starch. Therefore, analysing RS in digestion residues may be interpreted as characterization method, indicating the highly resistant fraction. Different amounts of RS were found because of additional non-starch material present in the samples as described above. Calculating RS recoveries based on TS led to $50 \mathrm{~g} / 100 \mathrm{~g}$ TS for the dynamically digested RTmd fractions, whereas after batch and in vivo digestion similar amounts (69 and $65 \mathrm{~g} / 100 \mathrm{~g}$ TS, respectively) were found. In contrast, clearly lower RS amounts were found in both HAM digestion residues (37 and $44 \mathrm{~g} / 100 \mathrm{~g}$ ) after dynamic and batch digestion, respectively.

In Fig. 1 the absorption of digestion products from the jejunum and ileum compartments during the dynamic experiment can be followed. The main digestion products found were maltose and maltotriose despite the fact that larger molecules are able to pass the hollow fiber devices as well. Starch digestion products up to DP3 were found when RTmd was being digested, whereas after HAM digestion minor amounts of maltotetraose (less than $0.1 \%$ of total digestible glucose) were detected as well. The kinetics of RTmd and HAM digestion differed considerably. The digestible fraction of RTmd was degraded completely after $4 \mathrm{~h}$, whereas degradation of HAM still continued after $4 \mathrm{~h}$. The lower amounts of degradation products collected during the first $4 \mathrm{~h}$ from HAM digestion, and the higher total amounts separated from the ileum compartment show that HAM under these experimental conditions is being digested more slowly compared to RTmd. The enzyme used for starch digestion in the dynamic model is a $\alpha$-amylase, therefore maltose is theoretically expected to be the smallest degradation product. As can be seen in Fig. 1, glucose was found as well. Glucose could originate from added compounds during the dynamic digestion experiment, such as bile and enzymes. Moreover, a small contamination of $\alpha$-amylase by AMG or $\alpha$-amylase containing small amounts of sideactivity cannot be excluded. The total amount of starch degradation products absorbed during the dynamic in vitro digestion experiment is slightly higher for HAM compared to RTmd. This is in accordance with a somewhat lower non-significant 

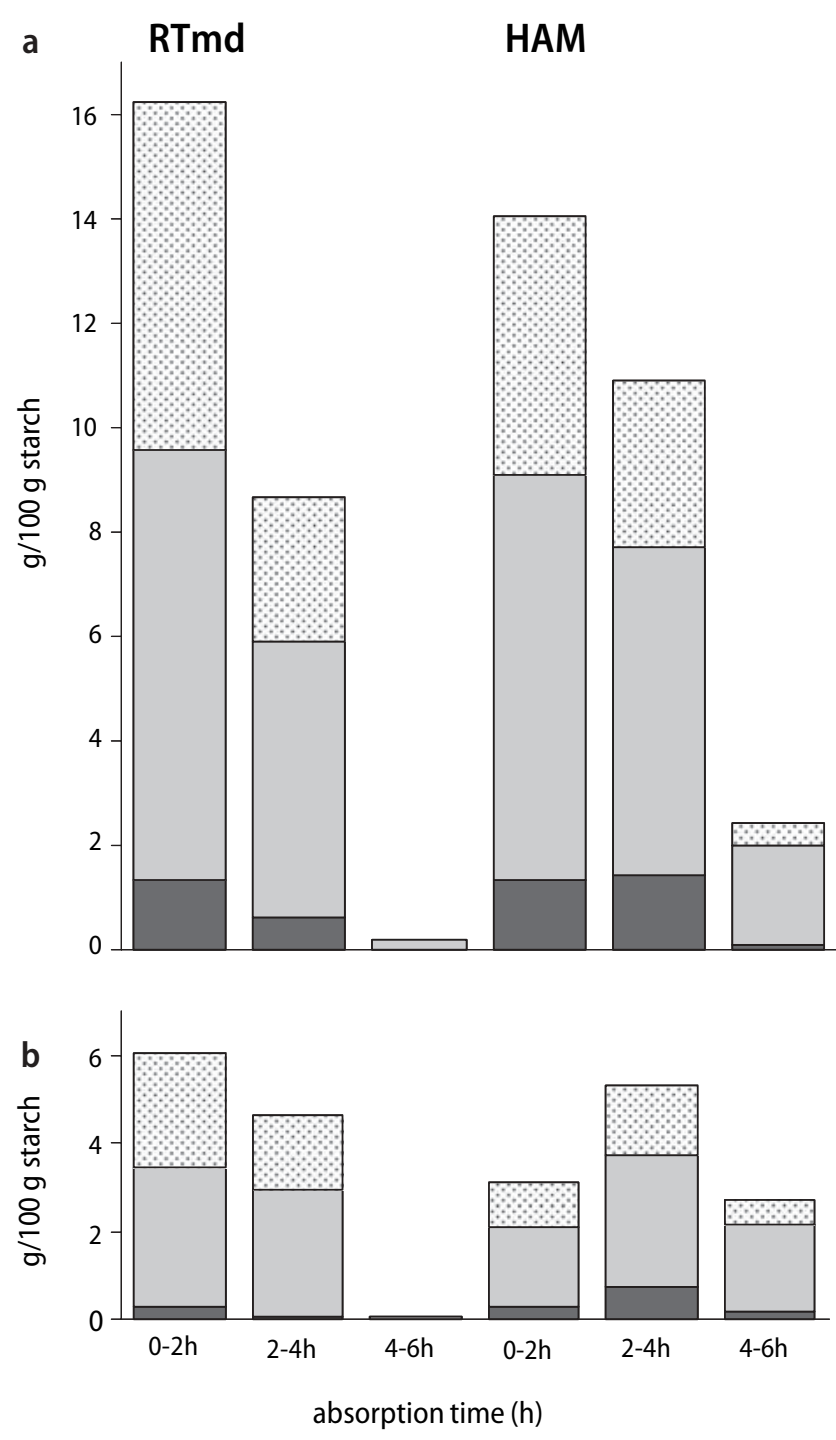

Glucose $\square$ Maltose $\square$ Maltotriose

Fig. 1 Digestible starch degradation products (up to DP3) of retrograded tapioca maltodextrins (RTmd), and high amylose maize starch (HAM) recovered from the jejunum (a) and the ileum (b) compartments

indigestible residue of HAM compared to RTmd as shown in Table 1.

\section{Characterization of digestion residues}

\section{X-ray diffraction}

X-ray diffraction patterns of RTmd and its dynamic, batch and in vivo digestion residues are presented in Fig. 2. RTmd and indigestible RTmd fractions all led to a typical A-type pattern. However, the diffraction patterns, with exception of RTmd dynamically digested,

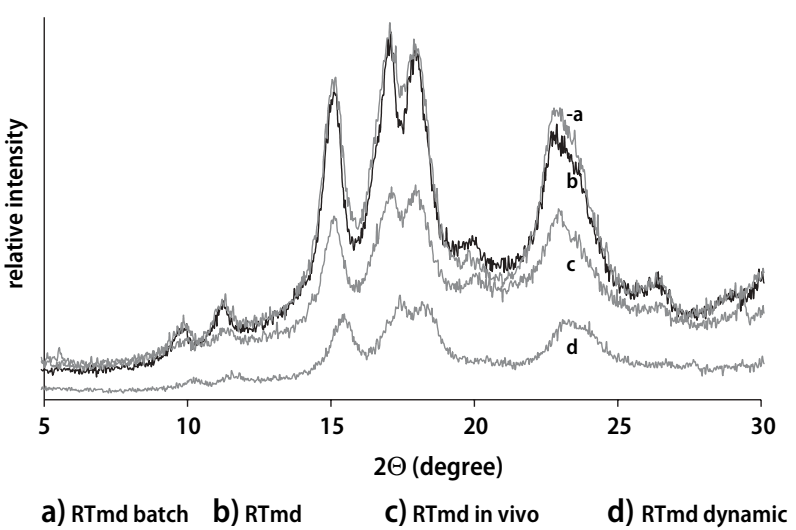

Fig. 2 X-ray diffraction patterns of retrograded tapioca maltodextrins (RTmd) and their digestion residues

exhibited an additional peak at $\theta 2=20^{\circ}$ which can be interpreted as $\mathrm{V}$-form due to the presence of amyloselipid complexes. RTmd and RTmd batch digested residues were very similar concerning their crystallinity. The only differences were the peaks at $2 \theta=20^{\circ}$, and $2 \theta=23^{\circ}$, which were slightly lower and higher, respectively in the batch digested sample. In contrast to RTmd and its batch digested fraction, dynamically digested RTmd showed no peak at $2 \theta=27^{\circ} \mathrm{C}$ and no pronounced double peak at $2 \theta=17^{\circ}$ and $2 \theta=18^{\circ}$ (both typical for A-type structure), which indicate a loss in crystallinity due to digestion. The in vivo digestion residue showed an additional peak at $2 \theta=29^{\circ}$; this peak could be due to additional structures of proteins or lipids present in this sample.

HAM containing samples led to lower crystallinity compared to RTmd (Fig. 3), which correspond to Gerard et al. [18], showing that high amylose maize starches have low crystallinity. HAM and its digestion residues led to B-type pattern, although the peak at $2 \theta=20^{\circ}$ observed in HAM and batch digested HAM

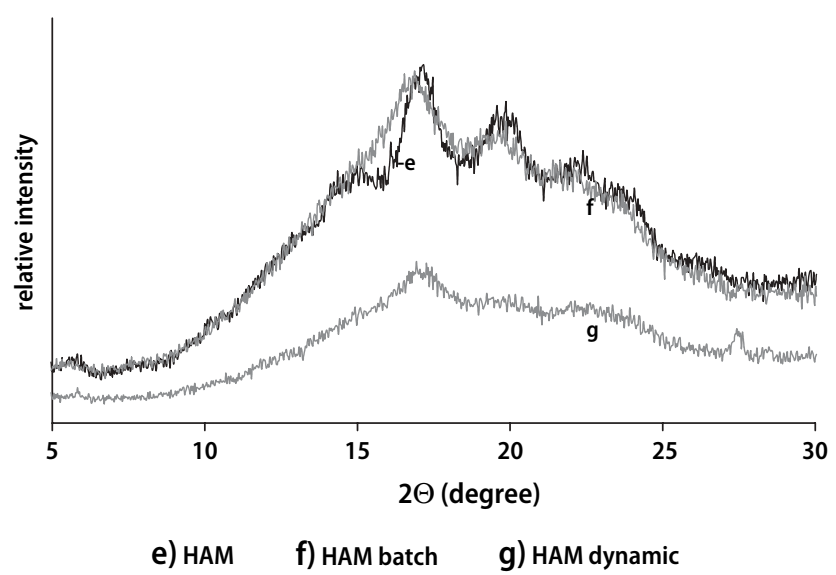

Fig. 3 X-ray diffraction patterns of high amylose maize starch (HAM) and their digestion residues 
indicated a mixture between B- and V-type which was described by Shi et al. [19] as well. Due to in vitro digestion of HAM and dynamically digestion of RTmd the peak $\theta 2=26^{\circ}$ disappeared which points to a loss in crystallinity. Similar as for RTmd (Fig. 2) the dynamic digestion led to higher changes in crystallinity compared to batch digestion.

\section{DSC}

The thermal behavior of the substrate melting was studied using DSC measurements. The results from the first and second heating are shown in Table 3. During the first heating, endothermic peak temperatures $\left(T_{\mathrm{p}}\right)$ turned out to be slightly lower for RS2 compared to RS3. The peak ranges within the RTmd and HAM containing preparations were found to be similar with exception of a slightly narrower peak of in vivo digested RTmd. Enthalpies were found to be lower for HAM containing preparations compared to RTmd containing preparations. The melting transition of RTmd was separated by an exothermic effect (not shown). Such a melting characteristic can be interpreted as partial melting (endothermic), followed by recrystallization (exothermic) and final melting (endothermic) [20,21]. This exothermic peak was not visible in the digestion residues, which indicates that due to digestion the fractions responsible for these rearrangements were removed. Within the RS3 fractions, in vivo digestion led to significantly lower enthalpies. Dynamic digestion of both preparations led to an exothermic peak when the sample was reheated (Table 3 ). This indicates that the reorganization of the crystalline structures takes place during the second heating. RTmd and its batch and in vivo digested fractions did not show any heat flow during the second heating (therefore not shown in Table 3), it can be interpreted that recrystallization was completed during cooling in these samples already. HAM containing samples, excluding the dynamically digested fraction, all led to an endothermic peak during reheating which indicates a reversible reconstruction of the amylose-lipid complexes [20].

\section{Discussion}

\section{Digestibility of RS preparations}

In vitro digestion models use a strictly standardized procedure based on an average human digestibility. Therefore standard deviations of in vitro experiments are expected to be low. In vitro obtained HAM fractions showed higher standard deviations compared to in vitro digested RTmd fractions. Possibly the molecular structures of HAM have been more sensitive to small differences in experimental conditions such as mechanical stress, temperature or $\mathrm{pH}$ between experiments.

RTmd in vitro digestion residues result in quantitatively similar RS fractions (Table 1 ) which are very well in accordance with $59 \mathrm{~g} / 100 \mathrm{~g}$ found in vivo after feeding RTmd to ileostomy patients [10]. In contrast, in vitro digestion using HAM as a substrate led to different amounts of RS. These differences may be explained by methodological variations in the in vitro models used, suggesting that HAM was more affected by the experimental conditions. During the dynamic digestion experiment the substrate is exposed to the movements of the flexible walls [8], in the batch model the slurry is stirred [7], and in the Megazyme RS method the sample is shaken [16]. An effect of mechanical treatment on starch degradation of HAM but not of RTmd was shown by McCleary and Monaghan [22] before. They performed the Megazyme RS method comparing shaking and stirring. Stirring led to a higher digestibility of HAM, whereas RTmd did not show any differences. In the present study however, stirring applied in the batch model led to a lower digestibility compared to shaking as used in the Megazyme RS method. This shows that apart from
Table 3 Average results $(n=3)$ from differential scanning calorimetry (DSC) for retrograded tapioca maltodextrins (RTmd) and high amylose maize starch (HAM) and their digestion residues.

$T_{0}$ : onset temperatures $\left({ }^{\circ} \mathrm{C}\right)$,

$T_{\mathrm{p}}$ : peak temperatures $\left({ }^{\circ} \mathrm{C}\right)$,

$T_{\mathrm{c}}$ : completion temperatures $\left({ }^{\circ} \mathrm{C}\right)$,

$\Delta \mathrm{H}$ : transition enthalpy $\pm \mathrm{SD}(\mathrm{J} / \mathrm{g})$

(endothermic if nothing else stated)

\begin{tabular}{llccr}
\hline Sample & $T_{0}\left({ }^{\circ} \mathrm{C}\right)$ & $T_{\mathrm{p}}\left({ }^{\circ} \mathrm{C}\right)$ & $T_{\mathrm{c}}\left({ }^{\circ} \mathrm{C}\right)$ & $\Delta \mathrm{H}(\mathrm{J} / \mathrm{g})$ \\
\hline First heating & & & & \\
RTmd $(n=2)$ & 60.7 & 112.8 & 140.4 & $9.0 \pm 1.1^{\mathrm{a}}$ \\
RTmd dynamic & 58.4 & 108.6 & 132.4 & $9.1 \pm 0.2^{\mathrm{a}}$ \\
RTmd batch & 61.8 & 109.1 & 136.4 & $10.8 \pm 2.0^{\mathrm{a}}$ \\
RTmd in vivo & 58.4 & 105.0 & 123.5 & $5.2 \pm 1.1^{\mathrm{b}}$ \\
HAM & 61.8 & 96.9 & 110.0 & $2.6 \pm 0.3^{\mathrm{c}}$ \\
HAM dynamic & 55.5 & 98.7 & 127.0 & $4.2^{\mathrm{a}} \pm 2.2^{\mathrm{c}}$ \\
HAM batch $(n=2)$ & 59.9 & 91.5 & 110.5 & $3.6 \pm 0.7^{\mathrm{c}}$ \\
Second heating & & & & \\
RTmd dynamic & 79.7 & 87.9 & 144.0 & $2.4 \pm 0.4$ \\
HAM & 60.0 & 88.2 & 109.6 & $2.7 \pm 0.9^{\mathrm{d}}$ \\
HAM dynamic & 84.9 & 94.2 & 146.1 & $* 2.8 \pm 0.2^{\mathrm{d}}$ \\
HAM batch & 59.8 & 83.2 & 101.9 & $0.9 \pm 0.0^{\mathrm{e}}$ \\
\hline
\end{tabular}

*Exothermic, means with the same letters are not significantly different (Tukey $P<0.05$ ) 
mechanical forces, also other factors may have a significant impact on starch degradation. For example, different amounts and types of enzymes, their accessibilities to the substrate as well as the formation of the enzyme-substrate complexes could have a larger influence on starch degradation of HAM compared to RTmd using in vitro digestion models. Furthermore, the incubation time during the dynamic digestion is nearly twice as long compared to the batch digestion and over four times as long in the Megazyme RS method. As pointed out in Fig. 1, 99\% of in vitro digestible RTmd fractions were degraded after $4 \mathrm{~h}$, whereas only $86 \%$ of those of HAM were digested at this stage. If the dynamic digestion experiment would have been stopped after $4 \mathrm{~h}$ (the duration of the batch digestion experiment is $3.75 \mathrm{~h}$ ), the remaining indigestible fraction of HAM would be identical to that obtained by the batch in vitro digestion. Whether a prolongation of the batch incubation would lead to HAM fractions similar to those obtained with the dynamic model, remains to be elucidated.

The analytical RS contents found in the dynamically digested residues was lower in both preparations when compared to the batch digested fractions (Table 2). This leads to the assumption that in the dynamic approach starch structures are attacked to a higher extent compared to the batch model. Nevertheless, the amount of TS recovered after digestion of RTmd was not affected, whereas the TS recovery of HAM possibly was influenced by the different amount of analytical RS present in the sample; the results strongly indicate that structural features play an important role in digestibility.

\section{Structural aspects of the RS preparations}

\section{X-Ray diffraction}

A mixture between A- and V-type structures for RTmd, and its in vivo digestion residues (Fig. 2) was found by Pohu [12] as well. The tightly packed A-type structure is known to be very heat stable. Shamai et al. [23] investigated RS3 fractions from high amylose maize, maize flour and wheat starch, respectively. They found that RS3 produced at low retrogradation temperatures of $40^{\circ} \mathrm{C}$ lead to B-type patterns, whereas incubation at $95^{\circ} \mathrm{C}$ produced a mixture of $\mathrm{A}$ - and V-type polymorphs. This is in contrast to the present study, where RS3 produced from long chain tapioca maltodextrins was found to consist of a mixture between A- and V-type crystal structures, even though rather low retrogradation temperatures of $52-54^{\circ} \mathrm{C}$ were applied [11]. This shows that the structural behavior of RS3 cannot be predicted without knowing the exact process parameters and these parameters are rarely disclosed, particularly in patents.

HAM led to a mixture of B- and V-type polymorphs, which is characteristic for native high amylose maize starch, as shown by several research groups [23-25]. The dynamically digested fraction did not show the V-type characteristic peak at $2 \theta=20^{\circ}$. This peak is known to represent amylose-lipid complexes. It is possible that the extra-addition of lipase in the dynamic digestion model was able to degrade lipids present in HAM and RTmd to an extent where no complexation was possible anymore. During batch digestion no additional lipase was used since this enzyme is present in the pancreatin preparation. In earlier experiments it has been shown that the $\mathrm{pH}$ optimum of the pancreatic lipase is approximatively 9 [26]. Therefore, the incubation at $\mathrm{pH} 6.9$ in the batch model is expected to lead to a small lipid degradation only. This could explain that the peak at $2 \theta=20^{\circ}$ was only reduced during batch procedure, but disappeared using the dynamic digestion model. In vivo digested RTmd showed amylose-lipid complexes (Fig. 2) as well. However, it can not be stated whether the lipid degradation in the dynamic model was more efficient compared to in vivo digestion because dietderived lipids led to a much higher lipid content before in vivo digestion (results not shown). The X-ray patterns of RTmd and HAM samples indicate that the molecules within the crystals are not packed in the same way which leads to different functional properties of HAM, and RTmd. Batch digestion of both preparations did not lead to a distinct change in crystallinity. However, in vivo digestion caused slightly broader, and dynamic digestion distinctly broader diffraction peaks, which indicate the presence of either imperfect or relatively small crystallites [27]. The latter could have occurred due to the mechanical stress which underlines that the dynamic model attacks the starch structures more strongly.

\section{DSC}

Endothermic transitions of the investigated samples took place at temperatures which are known to destroy the amylose-lipid complexes. At lower temperatures $\left(45-60^{\circ} \mathrm{C}\right)$ no peak was detected indicating that amylopectin is neither present in RTmd nor in HAM [20]. RTmd in vivo digested showed the lowest peak maximum and enthalpy of all the digestion residues. This may be due to the higher amount of non-starch material in the sample (Table 1) which may compete for water binding and thus reduce the starch swelling.

Dynamically digested HAM showed a slightly broader endothermic peak compared to the other samples which indicates a broader polydispersity [28]. This observation coincides well with the state- 
ment that starch is degraded differently, and probably more strongly in the dynamic model. In vitro digestion of HAM led to slightly but not significantly higher enthalpies, especially when dynamically digested. Cooke et al. [27] suggested that the enthalpy of gelatinization primarily reflects the loss of the double helical order which could occur to a higher extent during dynamic digestion.

\section{Conclusions}

The results of this study show that both, a simple and a more sophisticated in vitro digestion model and the ileostomy study led to similar amounts of starch escaping digestion when using a RS3 containing carbohydrate source as substrate. In contrast, when the two in vitro digestion models were compared with a RS2 containing preparation, different amounts of indigestible starch were found. It is not possible to predict which model simulated the in vivo RS2 frac- tion more precisely. The more sophisticated in vitro digestion model attacked starch probably more strongly compared to the batch in vitro digestion model using an RS2 and an RS3 containing substrate. This was confirmed by differences in crystalline fractions and structural rearrangements as measured by X-ray and DSC measurements. These structural differences, caused by different experimental parameters influenced starch digestibility of HAM but not of RTmd. The behavior of other RS preparations in the two in vitro models described here can not be deduced from the obtained results and has to be examined case by case.

Acknowledgements The authors wish to thank Karin Wehrmüller, Nausikaa Morenzoni and Karin Hotz for carrying out chemical analyses and batch in vitro digestion experiments, for technical assistance Jeffrey van Overeem using the dynamically model, Lydia Zehnder using X-Ray, Melanie Tietz using DSC and Béatrice Conde-Petit for physicochemical interpretation. The financial support by TNO Quality of Life and Cerestar-Cargill is gratefully acknowledged.

\section{References}

1. Englyst HN, Kingman SM, Hudson GJ, Cummings JH (1996) Measurement of resistant starch in vitro and in vivo. $\mathrm{Br}$ J Nutr 75:749-755

2. Baghurst PA, Baghurst KI, Record SJ (1996) Dietary fibre, non-starch polysaccharides and resistant starch: a review. Food Aust 48:S3-S35

3. Woo KS, Seib PA (2002) Cross-linked resistant starch: preparation and properties. Cereal Chem 79:819-825

4. Perrin P, Pierre F, Patry Y, Champ M, Berreur M, Pradal G, Bornet F, Meflah $\mathrm{K}$, Menanteau J (2001) Only fibres promoting a stable butyrate producing colonic ecosystem decrease the rate of aberrant crypt foci in rats. Gut 48:5361

5. Smith JG, German JB (1995) Molecular and genetic effects of dietary derived butyric acid. Food Technol 49:87-90

6. Champ MMJ (2004) Physiological aspects of resistant starch and in vivo measurements. J AOAC Int 87:749-755

7. Lebet V, Arrigoni E, Amadò R (1998) Digestion procedure using mammalian enzymes to obtain substrates for in vitro fermentation studies. Lebensm Wiss Technol 31:509-515

8. Minekus M, Marteau P, Havenaar R, Huisintveld JHJ (1995) A multicompartmental dynamic computer-controlled model simulating the stomach and small-intestine. ATLA Alternat Lab Animals 23:197-209
9. Champ MMJ, Martin LJ, Noah L, Gratas M (1999) Analytical methods for resistant starch. In: Cho S, Prosky L, Dreher $M$ (eds) Complex carbohydrates in foods. Marcel Dekker, Inc., NY, USA, pp 169-187

10. Brouns F, Andersson H, Arrigoni E, Brummer RJ, Fässler C, Kettlitz B, Langkilde AM, van Nieuwenhoven M, Philipsson H, Amadò R (2006) Physiological and metabolic properties of a novel type 3 Resistant Starch. J Agric Food Chem (Accepted)

11. Kettlitz B, Coppin J, Röper H, Bornet F (2000) Highly fermentable resistant starch. In: Cerestar Holding B.V., United States Patent No. 6043229

12. Pohu A (2002) Formation des amidons résistants au cours des traitments thermiques et enzymatiques. Dissertation. Universite de Nantes, France

13. Jörger F (1998) Digestibility and fermentability of differently processed wheat products. Doctoral Thesis No 12805. ETH, Zurich, Switzerland

14. McCleary BV, Gibson TS, Mugford DC (1997) Measurement of total starch in cereal products by amyloglucosidasealpha-amylase method: collaborative study. J AOAC Int 80:571-579

15. Boehringer (1984) In: Bestimmung von Glucose. Methoden der enzymatischen Lebenmittelanalytik mit Einzelreagentien. Boehringer $\mathrm{GmbH}$, Mannheim, Germany
16. Mc Cleary BV, McNally M, Rossiter P (2002) Measurement of resistant starch by enzymatic digestion in starch and selected plant materials: collaborative study. J AOAC Int 85:1003-1111

17. Lebet V, Schneider H, Arrigoni E, Amadò R (1994) Kritische Beurteilung der Proteinbestimmung nach der Methode von Kjeldahl aufgrund der Aminosäurenanalyse. Mitt Gebiete Lebensm Hyg 85:46-58

18. Gerard C, Colonna P, Buleon A, Planchot V (2001) Amylolysis of maize mutant starches. J Sci Food Agric 81:1281-1287

19. Shi Y-C, Capitani T, Trzasko PT, Jeffcoat R (1998) Molecular structure of a low-amylopectin starch and other highamylose maize starches. J Cereal Sci 27:289-299

20. Biliaderis CG (1998) Structures and phase transitions of starch polymers. In: Walter RH (ed) Polysaccharid association structures in food. Dekker M, New York, pp 57-168

21. Biliaderis CG, Galloway G (1989) Crystallization behavior of amylose-V complexes - structure property relationships. Carbohydr Res 189:31-48

22. Mc Cleary BV, Monaghan DA (2002) Measurement of resistant starch. J AOAC Int 85:665-675

23. Shamai K, Bianco-Peled H, Shimoni E (2003) Polymorphism of resistant starch type III. Carbohydr Polym 54:363-369 
24. Quan YS, Hattori K, Lundborg E, Fujita T, Murakami M, Muranishi S, Yamamoto A (1998) Effectiveness and toxicity screening of various absorption enhancers using Caco-2 cell monolayers. Biol Pharm Bull 21:615620

25. Sievert D, Czuchajowska Z, Pomeranz Y (1991) Enzyme-resistant starch. III. X-Ray diffraction of autoclaved amylomaize VII starch and enzyme-resistant starch residues. Cereal Chem 68:86-91
26. Tschäppät $S$, Arrigoni E, Amadò $R$ (1999) Development of an in vitro digestion method for fat rich food products. In: Lásztity $\mathrm{R}$, Pfannhauser W, Simon-Sarkadi L, Tömösközi S (eds) Functional Foods - A new challenge for the food chemists. EURO FOOD CHEM X. Publishing Company of TUB, Budapest, Hungary
27. Cooke D, Gidley MJ (1992) Loss of crystalline and molecular order during starch gelatinisation: origin of the enthalpic transition. Carbohydr Res 227:103-112

28. Moates GK, Noel TR, Parker R, Ring SG (1996) The effect of chain length and solvent interactions on the dissolution of the B-type crystalline polymorph of amylose in water. Carbohydr Res 298:327-333 